diskutiert. Ưber die chirurgischen Möglichkeiten zur Rekonstruktion der verschlossenen bzw. stenosierten Gefäßbahnen sollen Operationsskizzen informieren.

Anhand eines unausgelesenen, patho-anatomischen Sektionsgutes lassen sich Angaben über die Lokalisation und Häufigkeit, d. h. über Prädilektionsorte stenosierender Gefäßerkrankungen machen. Eigene Befunde werden mit den bereits im Schrifttum vorliegenden Daten korreliert. Auch die Pathomorphologie dieser Schäden wird anhand von Übersichtsbildern und histologischen Präparaten erläutert.

Durch die zunehmende Überalterung unserer Bevölkerung muß für die Zukunft mit einer erheblichen Zunahme charakteristischer und uncharakteristischer Störungen gerechnet werden. Deshalb sollte die Angiographie der Eingeweidearterien in stärkerem Maße und frühzeitiger als bisher zur Verbesserung der Erfolgsaussichten einer chirurgischen Therapie eingesetzt werden.

\title{
2. Erkennung und Behandlung von Verbrennungen
}

\section{K.-H. GRözINGER}

Chirurgische Universitätsklinik Heidelberg

Einteilung der thermischen Verletzungen nach Ursachen, Prognose, Oberflächen- und Tiefenausdehnung. Außer den lokalen Schädigungen spielen beim Hitzetrauma distante Organläsionen eine wichtige Rolle für den weiteren Verlauf. Nicht nur am Orte der Verbrennung, sondern auch in parenchymatösen Organen und in der übrigen unverletzten Haut ist die Mikrozirkulation durch arterielle Gefäßverschlüsse beeinträchtigt. In der Therapie ist die primäre Allgemeinbehandlung (Schmerz, Schock, Flüssigkeits-, Elektrolyt- und Eiweißersatz; zirkulatorische und respiratorische Uberwachung) ebenso wichtig wie die Lokalbehandlung. Hervorzuheben sind die Benutzung steriler Instrumente, das Anlegen von Schutzkleidung und die Raumhygiene. Die Verbrennungswunde soll offen oder mit filmbildenden Substanzen und chemokoagulierenden Pharmaka, besser mit fetthaltiger Gaze behandelt werden.

Im weiteren Verlauf sind lokal entzündungshemmende Verbände, Bäder, Krankengymnastik erforderlich. Neben optimaler Ernährung setzt eine erfolgreiche spätere plastische Versorgung die Beherrschung klinischer Probleme wie Stressulcus oder Nierenversagen voraus. In der Ausstellung werden neben historischen und zeitgenössischen Behandlungsverfahren wichtige Kriterien für eine adäquate und erfolgversprechende Therapie der Verbrennungen demonstriert. 